\title{
The effectiveness of a flood protection computer game for disaster education
}

\author{
Meng-Han Tsai ${ }^{1}$, Yu-Lien Chang ${ }^{2}$, Catherine Kao ${ }^{3}$ and Shih-Chung Kang ${ }^{3 *}$
}

\begin{abstract}
Background: Taiwan experiences typhoons annually and the heavy rain accompanies a typhoon often causes flooding and damage. Local decision-makers invest heavily in flood-prevention measures thus need to allocate resources wisely to minimize the destruction. As floods periodically cause tremendous damage to lives and property, passing on the learning of previous generations becomes important. Disaster education is therefore critical in the region. However, the current disaster education curriculum follows a traditional education methodology focusing on theoretical and conceptual knowledge and teaching. An alternative methodology is needed to motivate students and to deliver practical experience in disaster education.

Method: This study adopted a persuasive technology game-initiated-learning (GIL) and developed a Flood Protection game to understanding the following: (1) students' motivation for learning, (2) their ability to play the game, and (3) their focus on disaster topics. To evaluate their levels of motivation, students' reactions were analyzed and categorized into four specific expressions: discussion, question, laughter, and screaming. Students' game playing ability was analyzed and categorized into four stages: explore, aware, fluent, and known. Finally, the content of students' discussions and questions were analyzed to assess their interest in disaster education.

Results: Students' levels of motivation were found to be high (an average of 2.3 instances of discussion, 0.7 instances of question, 1.3 instances of laughter, and 0.8 instances of screaming were observed per minute during the game); their ability to play the game was sufficient (79\% of students completed the game having reached the "known" stage); and the content of their discussions and questions was closely related to disaster knowledge (73\% of the 256 discussions and questions related closely to flood and disaster knowledge).

Conclusions: From this research, we find that GIL is a persuasive design in flood protection training. Students with low motivation in conventional learning environments were triggered after playing the game. It enhanced students' motivation to learn more about flooding. In short, the game does benefit flood disaster education, indicating that a well-designed game may promote students' motivation in disaster education and cause behavior change.
\end{abstract}

Keywords: Game-based education; Disaster education; Behavior model; Learning motivation; Flood protection

\section{Background}

Disaster education is critical for regions suffering from the threat of flooding. Since floods periodically cause tremendous damage to lives and property, it is important to transfer wisdom and painful experiences from generation to generation (Dufty 2008).

The human mindset is mostly formed from education received at a young age, disaster education must start from basic education in the younger generation. It is

\footnotetext{
* Correspondence: sckang@ntu.edu.tw

${ }^{3}$ Department of Civil Engineering, National Taiwan University, No. 1, Sec. 4, Roosevelt Road, Taipei 10617, Taiwan

Full list of author information is available at the end of the article
}

advisable to start training courses in high school because the students have already acquired basic knowledge of geography as well as hydrology and are old enough to accept challenging strategic courses. Therefore, it is necessary to develop an effective flood disaster education approach to meet the need.

\section{Literature review \\ Game-based for disaster education}

Usually disaster prevention training is costly both in terms of time and money because exercise units need 
full-scale disaster settings to ensure that simulations are close to reality. Alternatively, exercises are tailored to conventional classroom conditions; however, traditional learning is mostly paper-based and promotes one-way information transfer, and has yet to combine knowledge with action (Ericksen 1984). Schank (1995) proposed "learning by doing" as a superior approach to the design of learning modules. In light of this, analysis of the current method of promoting disaster education suggests that a computer game that simulates a disaster site could be an effective teaching tool; such a tool would be interactive and enable users to accumulate learning experiences through repeated trial and error.

New computer simulation technologies have offered many flexible ways to carry out education and training for disaster prevention. These virtual environments with real disaster scenarios can be included in a serious game and can combine the engaging nature and realism of a simulation with the cost-effectiveness of a paper-based classroom teaching session (Sanders and Rhodes 2007). These games can simulate the disaster scenario in normal emergency training room and provide various possibilities of instructing with regard to the learning of soft skills and behavior for communication (Haferkamp and Krämer 2010).

An effective game in education is a harmonization of fun and educational value (Prensky 2003). Thus people cannot ignore the existence of the game and its function and influence in education. Bringing games into the environment of training projects should be considered seriously. Game-based learning was recognized by many researchers as having great potential when compared to traditional teaching and learning methods. Many studies have stressed the value of applying well-developed tuition strategies and theories to the design of educational games to enhance game-based learning (O'Neil and Fisher 2004).

\section{Persuasive technology}

Persuasive technology is technology designed to change attitudes or behaviors of the users through persuasion and social influence (Fogg 2002). Fantasy, curiosity and challenge can contribute to the fun in games (Amory et al. 1999; Asgari and Kaufman 2010; Habgood et al. 2005; Sanders and Rhodes 2007; Squire 2003; Whitehill and McDonald 1993). By incorporating fun components into tuition and a system design that contains motivational, learning and interactive elements, gaming can become effective in educational practice and learning (Quinn 1994).

The Fogg Behavior Model (FBM) provides designers and researchers with a systematic way to think about the factors underlying behavior change. In the FBM, behavior is a product of three factors: motivation, ability and triggers (Figure 1). A target behavior will occur when a person has sufficient motivation, acquires the ability to perform the behavior, and finally is triggered to carry out the behavior. These three factors must take place simultaneously, otherwise the behavior will not occur (Fogg 2009). In FBM, there are three kinds of triggers: spark trigger, facilitator trigger and signal trigger.

A spark trigger is appropriate to promote motivation and cause behavior change. On the other hand, a facilitator trigger is suitable for a person who has high motivation but lacks ability. A signal trigger works best when people have both motivation and ability to perform the target behavior. The FBM can be used to identify the problems in persuasive systems that are unable to obtain the target outcomes. In these situations, the FBM helps people identify the elements of motivation, elements of ability, and the strategies used for triggering behavior systematically. It is advisable to use the FBM to evaluate a persuasive design.

\section{Methods}

This study integrated the concepts of persuasive technology and game-based education to develop a learning approach known as game-initiated-learning (GIL). We assume that a game is a "spark trigger" that enhances students' learning motivation for disaster-related knowledge. The purpose of this study is to trigger students' interest in disaster prevention issues through the game we have developed, and to provide a scenario that faithfully simulates reality, to help students to understand and learn about the decision-making process in response to actual disasters. In the game, students must react to the disaster with limited time and resources to understand the importance of disaster prevention. This further triggers their interest in spontaneous learning on disaster prevention, elevating their motivation to learn.

To proceed with our research, we designed and implemented a computer game for GIL, and designed an experiment to investigate GIL in flood protection education. It was expected that playing the game would develop students' ability to the point of reaching the "aware" stage of the FBM.

\section{Research questions and method}

We determine whether the game can play the role of the spark trigger, helping students evolve from low motivation and high ability zone (no-action zone) to high motivation and high ability zone (action zone). In this study, behavior change is defined as follows: students are actively engaged in the learning process, have a willingness to learn, and have strong intentions to gain more knowledge. Answering the questions requires rigorous experiment design. 


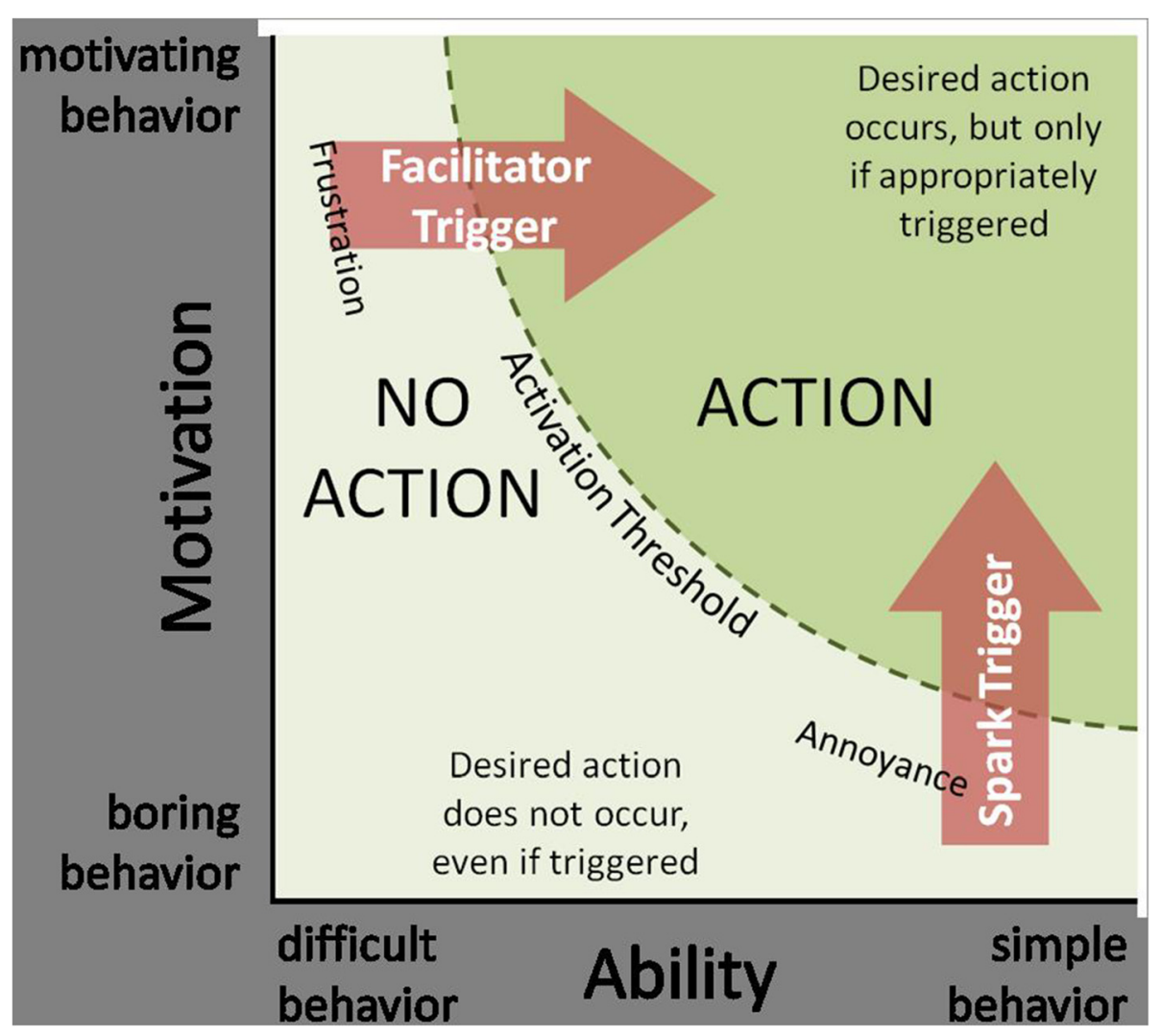

Figure 1 Fogg behavior model, adaptation of BJ Fogg's behavior (Fogg 2009).

The following are the methods used in analyzing the Motivation and Ability factors as well as the learning focus.

1. Motivation: The three core motivators for the motivation factor are Pleasure/Pain, Hope/Fear and Social Acceptance/Rejection. In this research we select Pleasure, Hope and Social acceptance as the motivators. In the GIL, students gathered together to play the game, naturally students express their social acceptance to each other when playing together. We need to prove that the other two core motivators were also relatively strong in order to conclude that motivation is high for the GIL.

2. Ability: The FBM uses six elements to define Ability: time, money, physical effort, brain cycle, social deviance and non-routine. In our research students are scheduled to attend the class and do not have to pay extra money for learning. They have no problems using the computer, and exhibit no socially deviant behavior. Therefore, our study should focus only on the element "brain cycle".

3. Learning focus: We shall summarize the students' audio or video records and the notes from the observers, during and after the game, into four categories: questions, findings, strategies and others; the results may be used to judge how the students' learning behaviors were affected.

\section{Game design and implementation}

The learning model, GIL, consists of three main steps: gaming, discussing and self-directed learning. In GIL, students can experience questions in the real world from gaming, discuss questions that they found in the game, and lastly, the instructors can teach related knowledge that is useful to solve the questions discussed (Tsai et al. 2014).

For this research, the computer game, Flood Protection, was designed and implemented. Figure 2 illustrates the game, each area has its own properties such as tax rates, funds, population, and capability against floods. During each level of the game, there are five waves of heavy rain which shall cause flood. Game players have to protect the three different areas in the city, depending on their thinking and strategies and prevent those areas from waves of floods by using selected engineering methods. The key to success relies on how well the students manage and allocate their resources. Special policies can increase the effectiveness of the resources in specific regions. After a certain number of floods, the total score will be evaluated and reflected on the screen. 


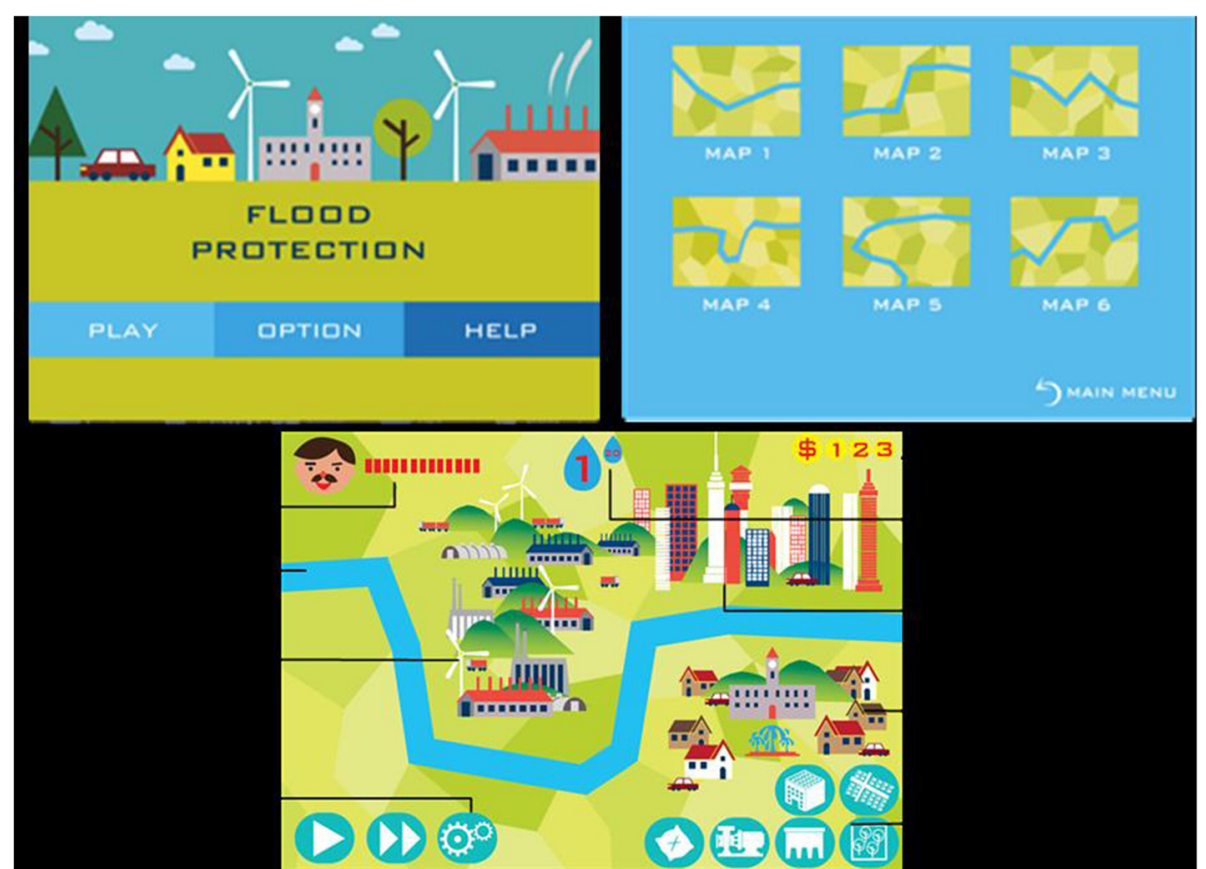

Figure 2 Flood protection game.

This game includes six different engineering methods for disaster mitigation: sand bags, pumps, dikes, retention parks, green roofs and green streets. Players can make use of varied engineering methods to protect from flooding. We designed six progressive levels, with different maps and difficulty levels, with a new engineering method added in each level. In the last level, level 6, all six engineering methods are unlocked for players to use. This setup allows them to have more choice in the decision making process. With increasing difficulty level, flood-prone areas become harder to protect. The players are expected to learn on a "trial and error" basis. This forces players to consider different strategies for manipulating the resources and the different engineering methods. The goal is not only for students to learn about flood protection through the game, but also for their learning motivation to be triggered through it. In other words, it is our hope that the game will both empower them to acquire knowledge about flood protection and encourage them to spontaneously seek to learn more about flood protection.

\section{Experiment design}

In this study, we investigated students' motivation and ability to play the GIL, with the aim of evaluating whether the game is capable of triggering a behavior change in the students. To this end, we designed an experiment including a test phase and measurement methods to assess motivation to learn, ability to play the game, and learning focus on disaster topics (Figure 3). In the pretest, we held an expert forum to ensure that the content of the game was suitable for disaster education and designed a questionnaire to understand students' background. We then conducted the field study, during which we observed and recorded students' reactions to playing the game along the dimensions of discussion, question, laughter, and screaming to analyze their motivation for learning, and categorized their ability as into the following phases: explore, aware, fluent and known. Their discussions and questions were recorded to analyze the focus of their learning. After the field study, we analyzed video recordings and observation notes to determine whether the game promoted students' motivation to learn about disaster prevention and whether it caused behavior changes. The following section describes the definitions of motivation, ability and learning focus used in this study.

In the framework of this study, students are considered to be motivated if they participate in the test, are interested in the game, and have a strong intention to learn about flood protection and other related areas of disaster management. We use "laughter" and "screaming" for the motivator Pleasure because laughter and screaming reflect the students' emotions of fun and happiness. We use "discussion" and "question" for the motivator Hope because both reactions can indicate students' desire and enthusiasm to learn; the students' expectation to win and their intention to play until the last moment are also indicators of Hope.

If the results of the investigation indicate that students' laughter and screaming become more frequent during 


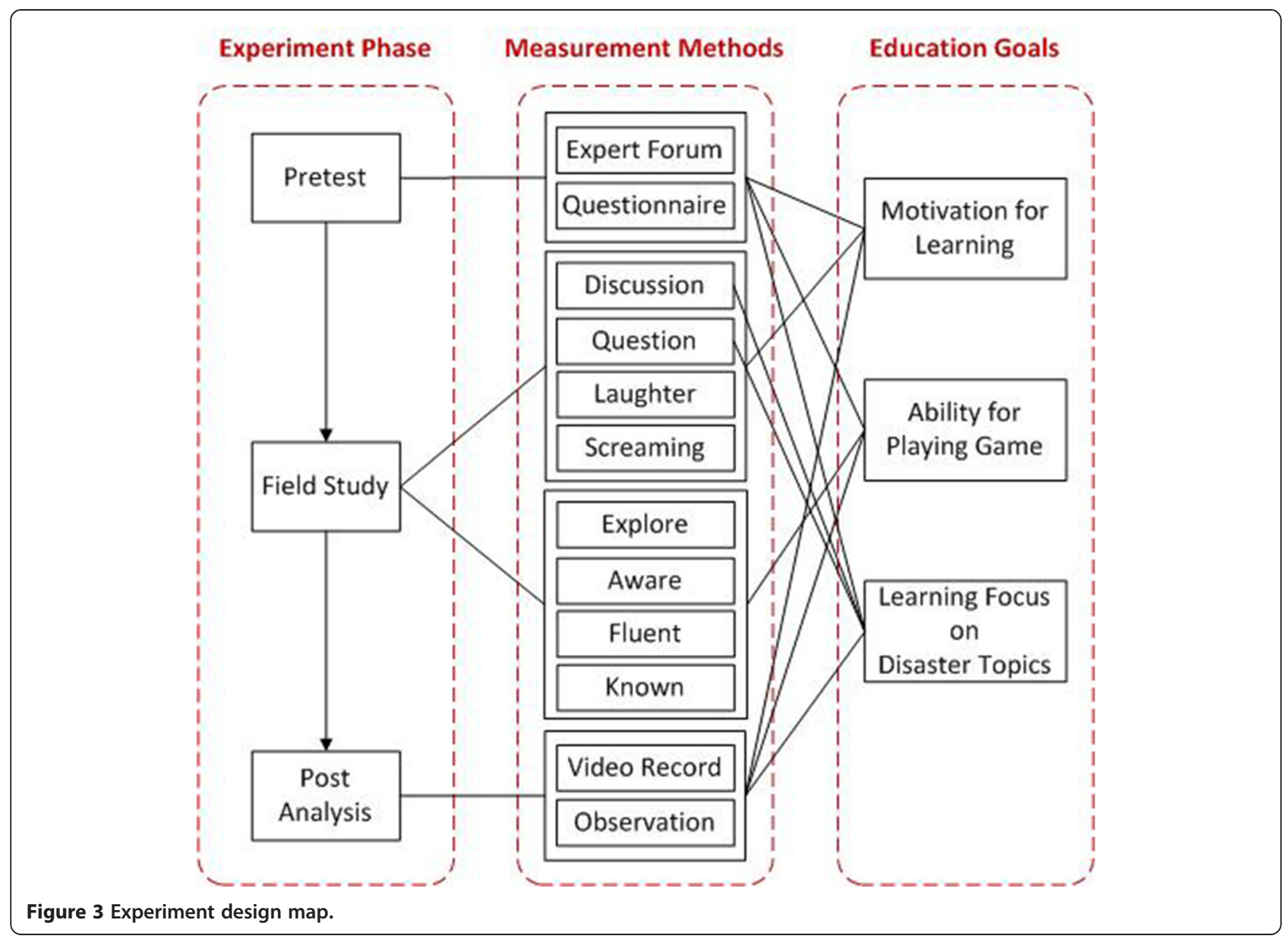

the game than before the game, and continue throughout the game, the motivator Pleasure is considered to be strong; the same may be applied to Hope. To gauge the prominence of the motivator of Social Acceptance, we observe students' interactions during game play to decide the strength of their social acceptance.

Ability is defined as students being able to play the game without having to learn in advance. In FBM, the ability factor has six elements: time, money, physical effort, brain cycles, social deviance and non-routine. Though we assume average high school students are at medium degree for the six elements, we still proceed with the evaluation of students' ability during the gaming. We shall analyze the video by categorizing students' abilities into four stages in each game: (1) Explore: the player moves the mouse on the screen at the beginning of each game, searching for the object of interest; (2) Aware: the player starts to notice new icons corresponding to engineering methods such as the sand bag, the pump, the dike, the green roof, or the green streets, and clicks them to try to use the new methods; (3) Fluent: the player can use the engineering methods fluently; (4) Known: the player can use the engineering methods without hesitation, demonstrating full knowledge of the underlying concepts.

In the game level 1, 2 and 3, we shall deem the game playing as well performed if students are able to play the game from "explore" through "known". In level 4, 5 and 6 , the game is more difficult because a higher level of engineering methods are used, so the game playing is deemed well performed if students are able to play the game from "explore" through "fluent". If most students are able to play through the game, we may conclude that students' abilities are high.

Learning focus in this test is defined as the direction and concern of students' question and discussion. To evaluate the learning focus in GIL, we will summarize the students' audio records and observers' notes, during and after the game, into four categories: questions, findings, strategies and others; the counts and contents of the results shall be calculated and evaluated. From the statistic data we can classify the students' interest, concern and the knowledge are related to which discussion contents and questions. If the counts are large and the contents are highly correlated to the flood disaster, students' learning behaviors are well triggered. 
If the results of the evaluation indicate that the learners have strong motivation and high ability and the trigger is properly and timely functioned through the learning, we may deem that the student behavior change occurs and is well performed.

\section{Pretest}

A pretest is carried out to ensure the content in the game is suitable for disaster education. Our advisers are professors and experts from various disciplines; these include but are not limited to water pollution, green economics, water footprint, water management, architecture, civil engineering, and education. An expert forum allows the experts to discuss ideas of how to improve the educational tools. Giving the experts an opportunity to discuss amongst themselves may increase chances of generating new and more ideas as opposed to individual interviews. All advisers expressed great hopes for the project and commented that the educational tools developed are suitable for senior high school students. Before implementing the GIL, a preliminary test on the Flood Protection was carried out. Students were invited to play the game, each group had two observers. During the test we found some common problems, therefore we designed a semi-structured questionnaire (Figure 4) based on the finding. The questionnaire was used as a guide for the observers during the GIL implementation. The results of the note made by the observers may be analyzed as part of the experiment data.

\section{Field study}

We arranged a one-day campaign in Huwei Senior High School in South Taiwan. The purpose of the campaign was to test the GIL by conducting a lesson on flood protection. Participants consisted of 33 ordinary senior high school students: 7 male and 26 female. The testees were distributed across three grades: 2 were juniors, 6 were sophomores and 25 were freshmen. Participants were divided into 11 groups of 3 students (Figure 5(a)). According to the pretest questionnaire, out of all 33 students, 24 had never participated in courses related to water resources or flood and typhoon protection, and 31 had never participated in flood prevention drills.

We provided 12 desktop computers which were installed with Flood Protection and screen recording software (Morae). Desktop computers were 27-inch iMacs with $3.2 \mathrm{GHz}$ Intel quad core i5 processors and 1GB video RAM. Students used the mouse to control and interact with the game. The program Morae was installed on the computers to provide video recording data for observation and analysis of learning behavior. The

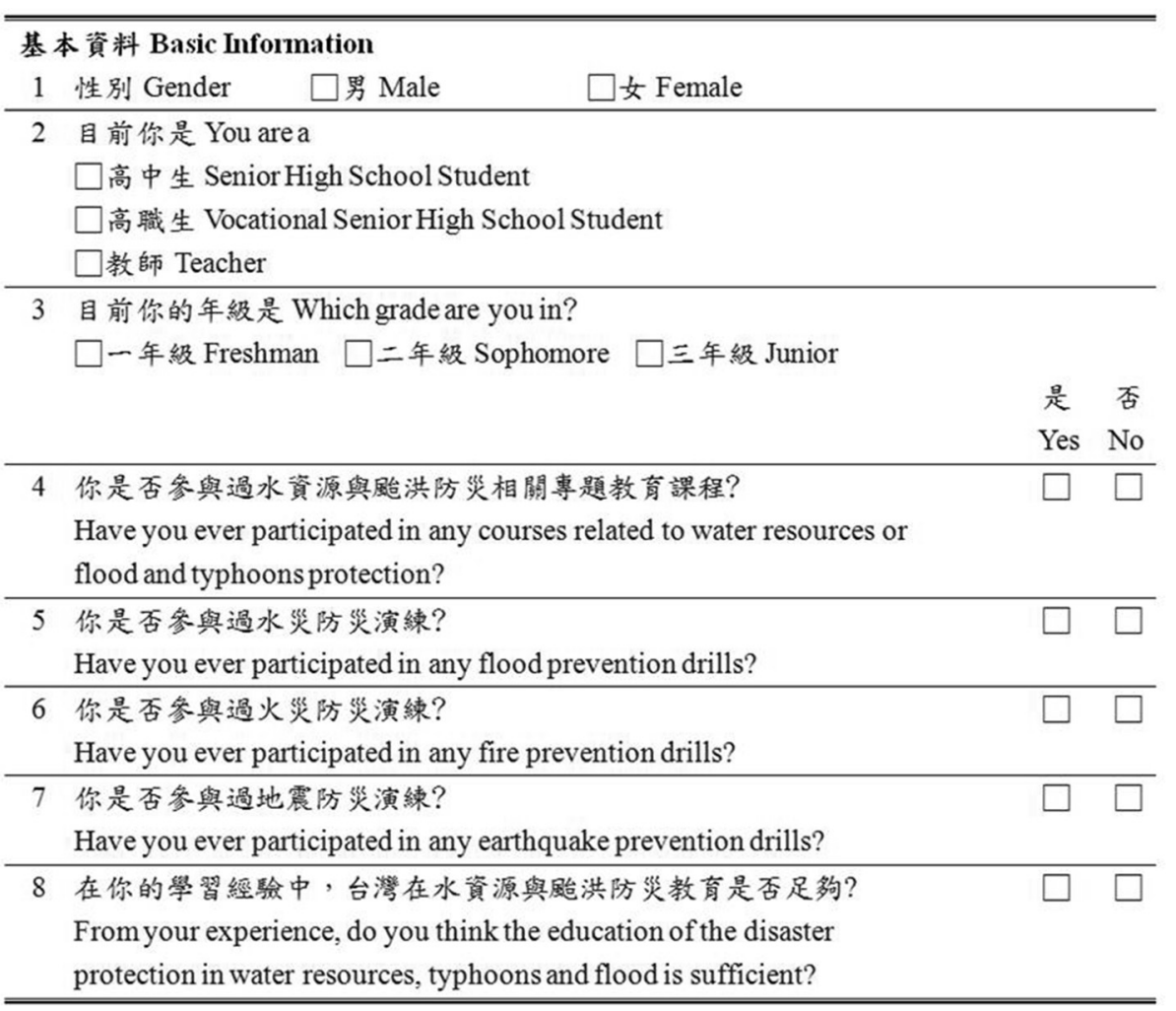

Figure 4 Questionnaire in pretest. 


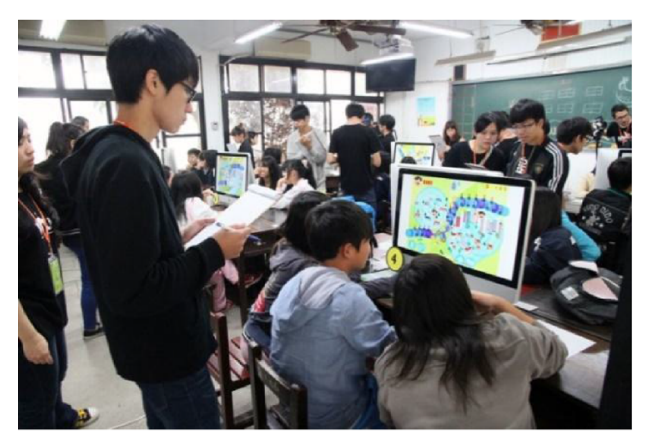

(a)

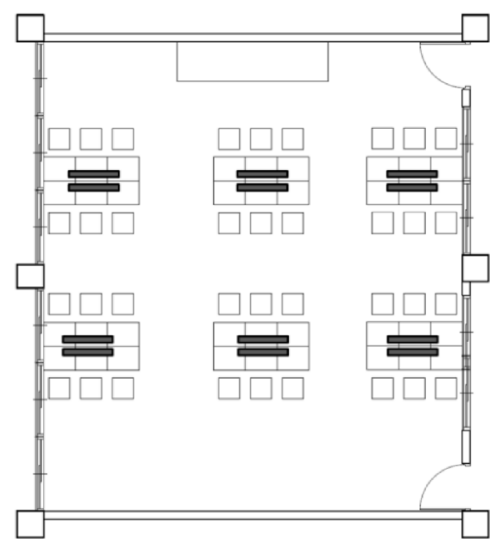

(b)

Figure 5 Field study. (a) Hu-Wei high school camp and (b) Arrangement of the test facility.

computers were arranged in a classroom that measured 8 meters by 9 meters (Figure 5(b)).

Before the students started playing the game, we did not provide any related engineering knowledge. The GIL started from students playing Flood Protection. The students then proceeded to discuss and share thoughts that might have arisen. And lastly, each student was given an eBook, displayed on an iPad, to browse through information, allowing them to gain knowledge by themselves and to engage in discussion and provide feedback within their respective groups. The content of the eBook was educational material on water-related disaster prevention, provided as a tool for exploration and reference during the teaching and learning of this subject matter.
The four themes of the eBook were water story, water Taiwan, water factory, and water city. The eBook covered issues including water resources operation and management, use of ecological engineering to achieve auto-purification by recycling and reusing water, and the current water rights distribution in Taiwan. The book was intended to offer participants the opportunity to gain further understanding of the principles and concepts in the game through reading and discussion.

The computer screen, mouse tracks and also players' voices and facial expressions were recorded during the game playing process. We also recorded the audio and video of the discussion and self-directed learning session and the observers took notes on the students' behavior

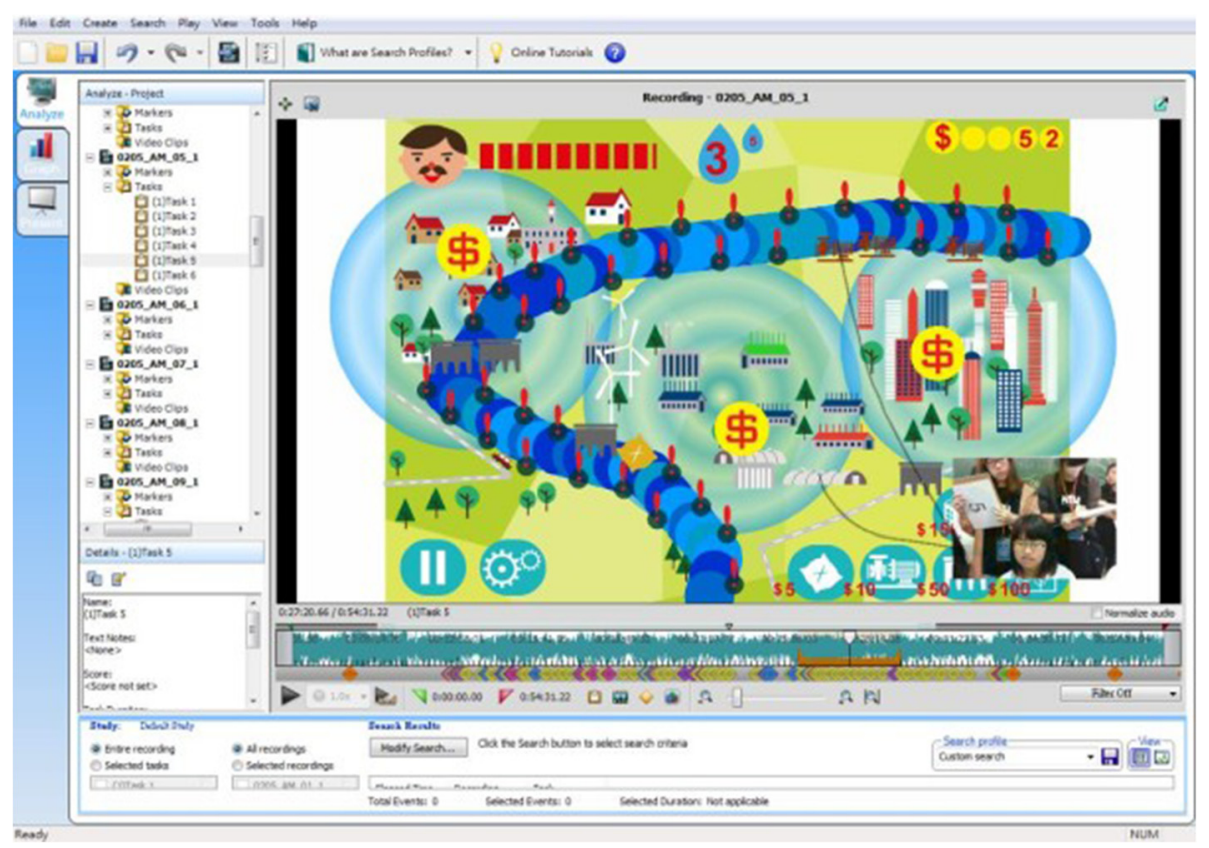

Figure 6 Video analysis tool. 
and all notable questions and discussion topics in the groups. After the campaign we sorted the collected data. The audio and video records as well as the observers' notes and records were analyzed and evaluated.

\section{Post analysis}

We used a software tool capable of recording, observing and analyzing the video game in this research. During the test we used it to record the playing process, including the computer screen, mouse track, students' facial expressions and voices. After the field test, we used it to analyze the data recorded (Figure 6). We first imported all records into MORAE Manager, then we used the function "define tasks" to define the games from level 1 to level 6 into six tasks. In each task we used function "define markers" to mark students' reactions and categorized them into four specific expressions: discussion, question, laughter and screaming, to determine their motivation. We then marked students' game playing and categorized their ability into four stages: explore, aware, fluent, and known; we finally analyzed the discussion contents and questions to see the relationship of students' interaction with our purpose: disaster education.

Table 1 Statistics of four expressions

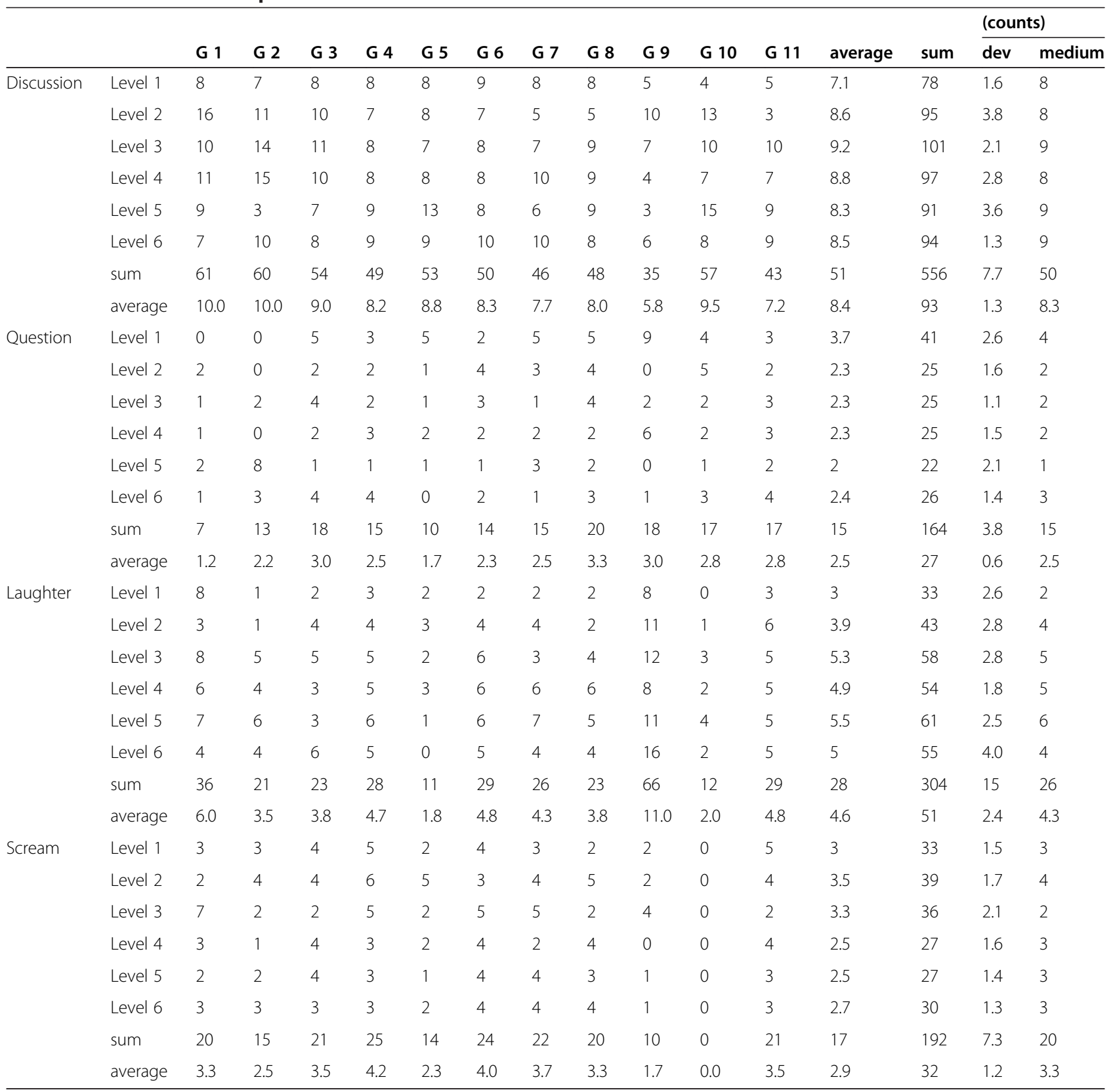


Table 2 Summarized notes

\begin{tabular}{|c|c|c|c|c|}
\hline \multirow[t]{2}{*}{ Category (Total counts) } & \multirow[t]{2}{*}{ Counts } & \multirow[t]{2}{*}{ Content } & \multicolumn{2}{|l|}{ Features } \\
\hline & & & knowledge & game \\
\hline \multirow[t]{15}{*}{ Question (105) } & 11 & Why do we have no money/why do we stop earning money? & $\bullet$ & \\
\hline & 11 & How to make money? & $\bullet$ & $\bullet$ \\
\hline & 11 & What is the symbol of the icon? & & $\bullet$ \\
\hline & 10 & Where do we put the sandbags? & $\bullet$ & $\bullet$ \\
\hline & 10 & Why do the pumps/ sandbags disappear? & $\bullet$ & $\bullet$ \\
\hline & 9 & Why does the flood occur? & $\bullet$ & \\
\hline & 8 & Which area can earn more money? & $\bullet$ & $\bullet$ \\
\hline & 8 & What is the blue circled area? Is it flooded area? & & $\bullet$ \\
\hline & 7 & What is the purpose of the park? Retention park? & $\bullet$ & \\
\hline & 5 & What is the purpose of green roofs & $\bullet$ & \\
\hline & 4 & What is the purpose of green streets? & $\bullet$ & \\
\hline & 4 & Where to put the green streets? & & $\bullet$ \\
\hline & 4 & Can we remove or sell the dikes? & $\bullet$ & $\bullet$ \\
\hline & 2 & Why this game is in English not Chinese? & & $\bullet$ \\
\hline & 1 & Is the city in Taiwan? & $\bullet$ & $\bullet$ \\
\hline \multirow[t]{14}{*}{ Finding (84) } & 9 & The sand bag could be used for a while. It will disappear. & $\bullet$ & $\bullet$ \\
\hline & 8 & The dike is a permanent protection construction. & $\bullet$ & \\
\hline & 8 & The blue circle is the flooded area. & & $\bullet$ \\
\hline & 7 & The index is happiness index. It seems very important! & & $\bullet$ \\
\hline & 7 & The less flooded area earns more money. & $\bullet$ & $\bullet$ \\
\hline & 7 & When we put it on correct location the flood is decreased! & $\bullet$ & \\
\hline & 6 & Commercial area earns money very quickly. & & $\bullet$ \\
\hline & 6 & When the happiness index disappears, the game fails. & & $\bullet$ \\
\hline & 5 & The pumps are very powerful! & $\bullet$ & $\bullet$ \\
\hline & 5 & The flood retention parks are expensive but very effective. & $\bullet$ & \\
\hline & 5 & We should pick up the money first & & $\bullet$ \\
\hline & 5 & Special region should be protected by special method. & $\bullet$ & \\
\hline & 4 & Green streets are expensive but very effective. & $\bullet$ & \\
\hline & 2 & Sand bag is very useful! & $\bullet$ & \\
\hline \multirow[t]{13}{*}{ Strategy (50) } & 7 & We should rescue the area where we can still earn money. & $\bullet$ & \\
\hline & 6 & Let's earn more money before starting the construction. & $\bullet$ & \\
\hline & 6 & Let' try to use new flood mitigation method. & $\bullet$ & \\
\hline & 5 & Let's use pumps in all areas! & $\bullet$ & \\
\hline & 4 & The whole river basin needs flood mitigation. & $\bullet$ & \\
\hline & 4 & We should mitigate the area of biggest flooded circle. & $\bullet$ & \\
\hline & 4 & Put dikes on the corners of the river! & $\bullet$ & \\
\hline & 3 & We should put the pumps near the flooded circle. & $\bullet$ & \\
\hline & 2 & We should put the sand bags with intervals. & $\bullet$ & \\
\hline & 2 & Put pumps on the corners of the river! & $\bullet$ & \\
\hline & 1 & Put sand bags on the corners of the river! & $\bullet$ & \\
\hline & 1 & We should put the pumps and sand bag with intervals. & $\bullet$ & \\
\hline & 5 & We should use multiple methods. & $\bullet$ & \\
\hline Others (17) & 6 & One mouse is not enough. & & $\bullet$ \\
\hline
\end{tabular}


Table 2 Summarized notes (Continued)

\begin{tabular}{lll}
\hline 6 & Hope we can play the game on line. \\
4 & I hope we can play together with a touch screen. \\
1 & I want to invent a sponge dike.
\end{tabular}

\section{Results}

This research yields three results. The first result is the analysis of the motivation by four coded specific expressions; the second result is an analysis of ability by four playing stages; the third result is the observation of learning experience.

\section{Results of motivation analysis}

To evaluate students' motivation, we watched the video records and coded students' interactions into four specific expressions: discussion, question, laughter and screaming during the playing process, which were the indices for the motivators - Hope and Pleasure.

Before the game started, students were almost quiet except few conversations which were not related to the lesson. Actually no specific expression was found. However they were active after the game started. Students asked question very often in the beginning of each level of the game and then followed by a series of discussions. The discussion continued for a while then the frequency reduced until the end of the game. Screaming occurred when new waves of flood came, and followed by the laughter later on.

The times of occurrence (counts) of the expressions from level 1 through level 6 for each group are listed in Table 1. The averages of total counts of the total six levels of the game are Discussion: 51, Question: 15, Laughter: 28, and Screaming 17; the medium of the four indices are Discussion: 50, Question: 15, Laughter: 26, and Screaming 20; the averages counts per minute during the game are: 2.3 discussion, 0.7 question, 1.3 laughter and 0.8 screaming. Statistical analysis revealed that the counts of discussion and screaming during the game did not vary much from beginning to end; questions were raised more in the beginning of the game and decreased in frequency as time in contact with the game increased; laughter increased along with time in contact with the game. Based on these results, we concluded that the gaming process triggered students' motivation.

\section{Results of ability analysis}

We watched the video records to see how well students played the game, which was assessed by the following method. We analyzed the video by categorizing four playing stages in each level of the game: explore, aware, fluent, known. We found that $79 \%$ of the students completed the game having progressed from the "explore" stage all the way to the "known" stage.

\section{Results of observations on learning focus}

Next we evaluated how well the behavior changed. We summarized the students' audio records and the notes from the observers, during and after the game, into four categories: questions, findings, strategies and others. From the audio records, we found that students had a lot of discussions about the game. They were interested in the flood protection methods, especially the green streets, green roofs, and retention park, which they were not familiar with and were reluctant to play during the game. Records from each observer also reflected that the students were very happy to play the game and ask a lot of questions about the game and the lessons. Table 2 shows the statistics of the summarized data. They had 105 questions, 84 findings, 50 strategies and 17 other issues. The discussion contents and questions are closely related to disaster knowledge: $73 \%$ of the 256 discussions and questions are about flood knowledge.

\section{Discussion}

Following is our finding on students' motivation, ability and learning focus. We also make a comparison between GIL and traditional learning to explain how game can trigger students' learning behavior.

\section{Finding}

The results of the field study were analyzed. We describe our finding on students' motivation, ability and learning focus as below:

1. Motivation: We use "laughter" and "screaming" for the motivator Pleasure, "discussion" and "question" for motivator Hope. We found that students were almost quiet before the game started, but the four specific expressions appeared very frequently during the game, indicating that students were active during gaming. We also found that all 11 groups had completed their games from level 1 through level 6 whether they succeeded or failed at each separate level of the game. Therefore, we could conclude that both motivators, Hope and Pleasure were very high. Meanwhile, judging from the whole gaming process students' exhibited social acceptance for one another. Based on the above analysis, we could conclude that students' motivations in the gaming process were triggered.

2. Ability: We could see in each level that students find the new engineering method easily and get familiar 
Table 3 Comparison between GIL and Traditional learning

\begin{tabular}{|c|c|c|c|}
\hline Factors & Elements of the factors & Traditional learning & GIL \\
\hline \multirow[t]{6}{*}{ Motivation } & Pleasure/Pain & Less motivating & Motivating \\
\hline & & Reading textbooks is less fun and pleasant. & $\begin{array}{l}\text { Playing Flood Protection, a game of edutainment, is a pleasure } \\
\text { Students' laughter and screaming reflect emotions of fun and } \\
\text { happiness. }\end{array}$ \\
\hline & Hope/Fear & Less motivating & Motivating \\
\hline & & $\begin{array}{l}\text { Anticipating much homework and many } \\
\text { memorizing tasks. }\end{array}$ & $\begin{array}{l}\text { Playing game is attractive. Students' discussion and question } \\
\text { represent the students' desire and anxiety to learn, }\end{array}$ \\
\hline & Social acceptance/Rejection & Less motivating & Motivating \\
\hline & & $\begin{array}{l}\text { Reading cannot enhance relationship with } \\
\text { others. }\end{array}$ & $\begin{array}{l}\text { Playing game enhance social acceptance with classmates and } \\
\text { teachers. }\end{array}$ \\
\hline \multirow[t]{6}{*}{ Ability } & Time & \multicolumn{2}{|c|}{ Simple: Students are scheduled to attend the class. } \\
\hline & Money & \multicolumn{2}{|l|}{ Simple: Students do not have to pay. } \\
\hline & Physical effort & \multicolumn{2}{|l|}{ Medium } \\
\hline & Brain cycle & \multicolumn{2}{|l|}{ Medium } \\
\hline & Social deviance & \multicolumn{2}{|l|}{ Simple } \\
\hline & Non-routine & \multicolumn{2}{|l|}{ Simple } \\
\hline
\end{tabular}

with the new method quickly. Most students were not only able to perform the games smoothly and successfully but also understood the game in the right way. There was no doubt from these results that the students' ability was strong.
3. Observation of learning focus: We found that students carried out a lot of discussions on the game. They were interested in the flood protection method, especially the green streets, green roofs, and retention parks, which they were not familiar

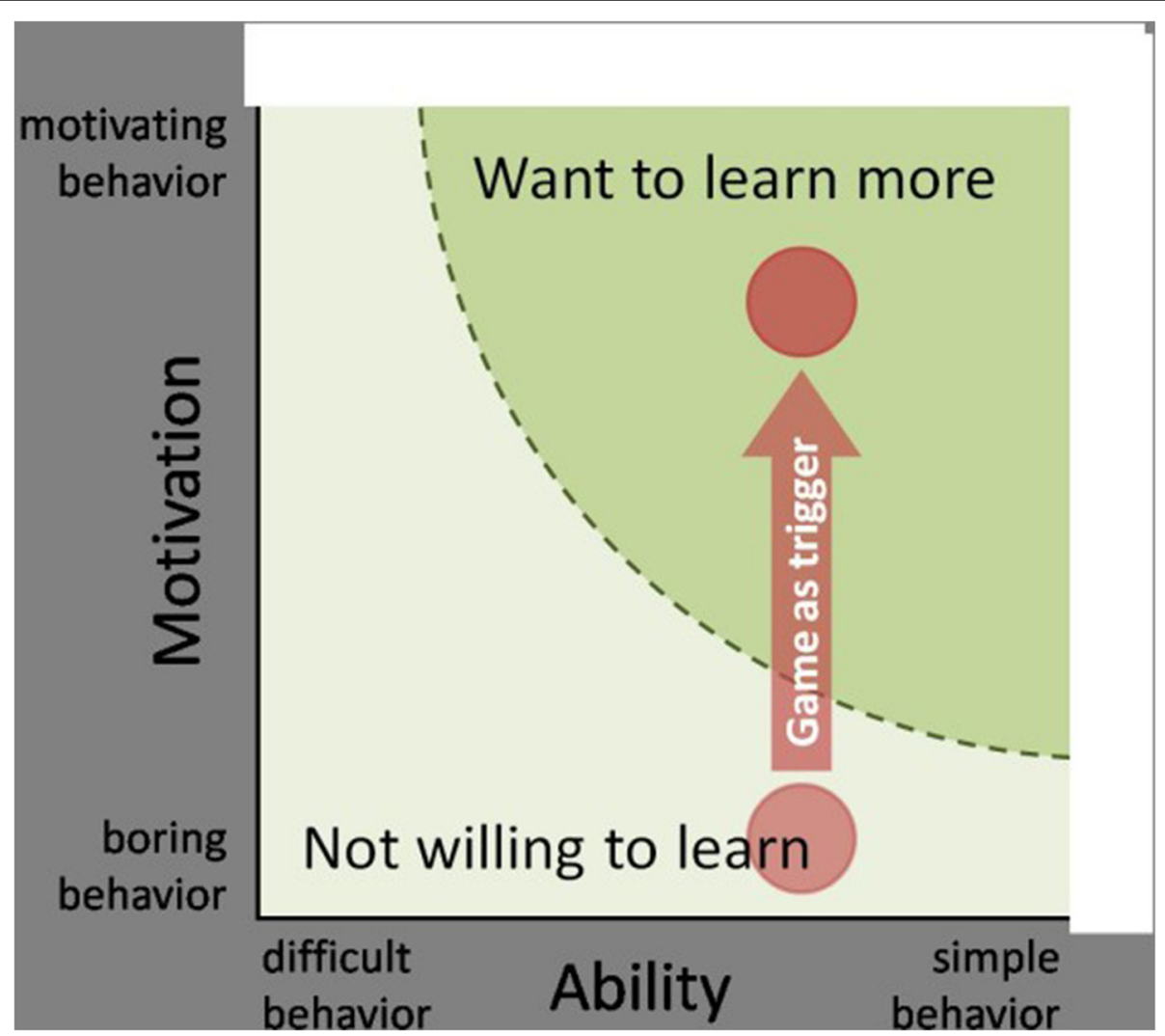

Figure 7 FBM for GIL. 
with and were reluctant to play during gaming. Records from each observer also reflected that students were happy to play the game and ask a lot of questions about game and the lessons.

\section{Comparison between GIL and traditional learning}

In this research, we investigated students' motivation and ability for the GIL aiming to find out whether game could trigger students' behavior change and how it differentiated from traditional learning. Table 3 shows the comparison between GIL and traditional learning in regard to Motivation and Ability. The three core motivators for the motivation factor and six elements for the ability factor in FBM were used as a guide to evaluate the motivation as well as ability.

With regard to motivation, in traditional learning, students are less motivated because textbooks are less fun and lack involvement (Hake, 1998), homework and extensive memorization discourage students to learn, and interactions between students are limited (Ericksen, 1984). In this case, students' motivators tend towards the opposites of pleasure, hope and social acceptance, resulting in decreased motivation to learn and in mere memorization without making connections to real-life applications (McKeachie, 1986). However, in our GIL-based study, students were encouraged to learn due to the pleasure they obtained from playing Flood Protection, a game of edutainment. Students' laughter and screaming reflected emotions of fun and happiness. Students were also stimulated by the game; their discussion and questions were indicative of their strong involvement in their own learning.

For ability we assume average high school students are at medium degree for the six elements. Our findings in this indicate that students' ability to gaming is strong.

Based on the above observations and analysis, students were triggered by the game and promoted to a high motivation level in the flood protection campaign. Their ability was high, which was normal for high school students. The trigger (Flood Protection) was effective and successful. During the discussions and self-directed learning, students' behavior revealed that they were actively engaged in the learning process, were willing to learn and had strong intentions to gain more knowledge.

Our findings indicate that students have low motivation and high ability in traditional learning environments. The game appears to be a spark trigger. When students engage with the GIL, their motivation appears to be sparked and heightened by the game, and the game seems to trigger students' behavior to learn more in the future (Figure 7).

\section{Conclusions}

We conclude that Flood Protection is a positive trigger in GIL. The four specific expressions: discussion, question, laughter, and screaming are valuable references for the motivators of hope and pleasure; the four stages approach can be used to analyze and evaluate the players' ability in the game; the observation of learning focus shows that students ask related questions and find strategies to fight the floods. From this research, we also find the GIL is a persuasive design in flood protection training. Students who have low motivation in conventional learning environment were triggered after playing the game. It has enhanced students' motivation to learn more about flooding. In short, the game does benefit flood disaster education, thus it indicates a well-designed game may promote students' motivation in disaster education and cause behavior change.

\section{Consent}

Written informed consent was obtained for the publication of this report and any accompanying images.

\section{Competing interests}

The authors declare that they have no competing interests.

\section{Authors' contributions}

MHT and YLC together developed the methodology and game. All authors together carried out the usability test, analyzed the results and drafted the manuscript. CK assisted the literature review and the usability test. SCK offered suggestion and guidance to the research. YLC and CK drafted the manuscript and MHT reviewed and revised it. All authors read and approved the final manuscript.

\section{Acknowledgments}

This research is supported by Taiwan's Ministry of Science and Technology (MOST) under contract 101-2515-S-002-002.

\section{Author details}

${ }^{1}$ Center for Weather Climate and Disaster Research, National Taiwan University, No. 1, Sec. 4, Roosevelt Road, Taipei 10617, Taiwan. ²Department of Interior Design, Hwa Hsia University of Technology, No. 111, Gongzhuan Rd, Zhonghe District, New Taipei 235, Taiwan. ${ }^{3}$ Department of Civil

Engineering, National Taiwan University, No. 1, Sec. 4, Roosevelt Road, Taipei 10617, Taiwan

Received: 15 October 2014 Accepted: 16 February 2015 Published online: 15 March 2015

\section{References}

Amory, A, Naicker, K, Vincent, J, \& Adams, C. (1999). The use of computer games as an educational tool: identification of appropriate game types and game elements. British Journal of Educational Technology, 30(4), 311-321.

Asgari, M, \& Kaufman, D. (2010). “Does fantasy enhance learning in digital games?". In D Kaufman \& L Sauvé (Eds.), Educational Gameplay and Simulation Environments: Case studies and lessons learned (pp. 84-95). Hershey, PA: IGl Global.

Dufty, N. (2008). A new approach to community flood education. The Australian Journal of Emergency Management, 23(2), 4-8.

Ericksen, S. (1984). The essence of good teaching: Helping students learn and remember what they learn. San Francisco: Jossey-Bass.

Fogg, BJ. (2002). Persuasive technology: using computer to change what we think and do. USA: Morgan Kaufmann.

Fogg, BJ (2009). "A behavior model for persuasive design." Proceedings of the 4th International Conference on Persuasive Technology. Claremont, California. April 26-29.

Habgood, MPJ, Ainsworth, SE, \& Benford, S. (2005). Endogenous fantasy and learning in digital games. Simulation and Gaming, 36(4), 483-498.

Haferkamp, N, \& Krämer, NC. (2010). Disaster readiness through education - Training soft skills to crisis units by means of serious games in virtual environments. Lecture Notes in Computer Science, 6383, 506-511. 
Hake, RR. (1998). Interactive-engagement vs traditional methods: a six-thousandstudent survey of mechanics test data for introductory physics courses. American Journal of Physics, 66, 64-74.

McKeachie, WJ. (1986). Teaching tips: A guidebook for the beginning college teacher. Lexington, MA: D.C. Heath and Company.

O'Neil, HF, \& Fisher, YC. (2004). "A technology to support leader development: computer games." Leader development for transforming organizations: Growing Leaders for Tomorrow (pp. 99-121). NJ: Lawrence Erlbaum Associates.

Prensky, M. (2003). Digital game-based learning. ACM Computers in Entertainment, 1(1), 1-4.

Quinn, CN. (1994). "Designing educational computer games". In K Beattie, C McNaught, \& S Wills (Eds.), Interactive multimedia in University Education: Designing for change in teaching and learning (pp. 45-57). Amsterdam: Elsevier Science BV.

Sanders, RL and Rhodes, GS (2007). "A simulation learning approach to training first responders for radiological emergence." Proceedings of the 2007 Summer Computer Simulation Conference, San Diego, California. July 16-19.

Schank, RC (1995). What We Learn When We Learn by Doing. (Technical Report No. 60). Northwestern University, Institute for Learning Sciences. Retrieved January 122014 from: http://cogprints.org/637/1/LearnbyDoing_Schank.html. Squire, K. (2003). Video games in education. International Journal of Intelligent Simulations and Gaming, 2(1), 49-62.

Tsai, MH, Wen, MC, Chang, YL, Kang, SC (2014). "Game-Based Education for Disaster Prevention." Al \& Society. doi:10.1007/s00146-014-0562-7.

Whitehill, BV, \& McDonald, BA. (1993). Improving learning persistence of military personnel by enhancing motivation in a technical training program. Simulation and Gaming, 24(3), 294-313.

\section{Submit your manuscript to a SpringerOpen ${ }^{\circ}$ journal and benefit from:}

- Convenient online submission

- Rigorous peer review

- Immediate publication on acceptance

- Open access: articles freely available online

- High visibility within the field

- Retaining the copyright to your article

Submit your next manuscript at $>$ springeropen.com 